\title{
Study of the critical behavior of antiferromagnetic thin films by computer modeling
}

\author{
S. V. Belim ${ }^{\dagger}$, E. V. Trushnikova \\ †sbelim@mail.ru \\ Dostoevskiy Omsk State University, 55a Mira ave., Omsk, 644077, Russia
}

\begin{abstract}
In this paper, the surface phase transitions in thin films described by the antiferromagnetic Ising model are studied by computer modeling. The Metropolis algorithm is used. Modeling is performed at various values of the ratio between the exchange integrals on the surface and in the bulk of the system, $R_{s}$. The difference of the exchange integral of the interaction between the surface spins and the first subsurface layer, $R_{S B}$, from the bulk value is also taken into account. The limiting cases of the $R_{S B}$ value are considered. Two order parameters are used. The first order parameter determines the antiferromagnetic order in the bulk of a system. It is calculated as the staggered magnetization of the spins located not on the surface. Its value is equal to the difference between the magnetic moments for two sublattices. For the study of the surface phase transition, the second order parameter is introduced. It is calculated as the staggered magnetization of the spins located on the free surface. In order to find the phase transition temperature, the bulk and surface Binder's cumulants are used. A computer experiment is performed for different values of film thickness ranging from 4 to 12 layers. The ratio between the exchange integrals varies from 0.5 to 2.0 . It is shown that the temperatures of the bulk and surface phase transitions are identical at all the ratios between the exchange integrals. The transition temperature grows with increasing ratio between the exchange integrals, $R_{S}$. The growth rate of the transition temperature depends on the thickness of a film and the $R_{S B}$ value. The difference of the exchange integral of the interaction between the surface layer and the first subsurface layer leads to more rapid growth of the transition temperature. For all the values of exchange integrals there is an intersection point of temperature curves for any thickness of a film.
\end{abstract}

Keywords: antiferromagnetics, thin films, phase transitions, critical phenomena.

\section{Introduction}

Surface magnetism is observed experimentally in both ferromagnetic and antiferromagnetic systems. In order to describe this phenomenon, the notion of surface magnetic energy was introduced [1], which enabled calculating the shift of the Curie temperature on the free surface of semibounded ferromagnetic systems with respect to the bulk value. On the basis of a phenomenological approach, a phase diagram was built for semi-bounded systems with a free surface $[2,3]$. Three phases may be observed in a system, which are related to the ordering of the surface and bulk spins: a disordered phase (SD/BD), a surface-ordered bulkdisordered phase $(\mathrm{SO} / \mathrm{BD})$ and a surface-ordered bulkordered phase (SO/BO).

There are three types of phase transitions that are possible in a system. The transition from $\mathrm{SD} / \mathrm{BD}$ to $\mathrm{SO} / \mathrm{BD}$ is called a surface phase transition, from $\mathrm{SO} / \mathrm{BD}$ to $\mathrm{SO} / \mathrm{BO}-$ an extraordinary phase transition, from $\mathrm{SD} / \mathrm{BD}$ to $\mathrm{SO} / \mathrm{BO}-$ an ordinary or bulk phase transition. The three lines of phase transitions intersect at a tricritical point; the phase transition at this point is called a special one. The critical phenomena in the vicinity of the lines of these transitions in the framework of the field-theory approach are described in the papers $[4,5,6]$.

It was experimentally observed in a number of studies that the surface phase transition temperature $T_{s}$ was higher than the bulk phase transition temperature $T_{c}$ $\left(T_{s}-T_{c}=15 \mathrm{~K}\right)$ in polycrystalline Gd [7]. However, cases were experimentally observed where surface ordering occurred at a lower temperature. For example, for the antiferromagnetic macroscopic $\mathrm{Fe}_{3} \mathrm{BO}_{6}$ crystal, $T_{c}-T_{s}=8.5 \mathrm{~K}$, and for the $\mathrm{Fe}_{3} \mathrm{BO}_{6}$ crystal [8], $T_{c}-T_{s}=5 \mathrm{~K}$.

The difference in the values of the surface and bulk exchange integrals was calculated from the first principles in the papers $[9,10]$, and experimentally verified in $[11,12]$. In the paper [13], on the basis of the first-principles calculations it was shown that for $\mathrm{FeO}$ the surface energy depends linearly on the chemical potential. In the paper [9], the first-principles calculations for $\mathrm{Gd}$ revealed that the distance between atoms in the bulk of a crystal equals $3.52 \mathrm{~A}$, whereas on the surface it equals $3.64 \mathrm{~A}$, which leads to a difference in the values of exchange integrals, being $J_{S}=1.25$ and $J_{B}=1.51$, respectively, and consequently, their ratio is $R=J_{S} / J_{B}=0.83$.

The computer modeling of both ferromagnetic and antiferromagnetic semi-bounded systems $[14,15,16]$ showed that the surface phase transition temperature is higher than the bulk one when the ratio of the surface exchange integral to the bulk exchange integral is $J_{S} / J_{B}>1.55$. At a value of $J_{S} / J_{B}=1.55$, a special phase transition is observed. The phase diagram here corresponds to the one predicted by the phenomenological theory. The possibility of the state where the bulk temperature is higher than the surface one for the ferromagnetic Ising model was demonstrated by computer 
modeling in the papers $[17,18]$. Here, a surface-disordered bulk-ordered phase $(\mathrm{SD} / \mathrm{BO})$ is observed in the phase diagram of the substance. The existence of such a phase is possible only when the ratio between the exchange integrals is $J_{S} / J_{B}<1$. In this case, the phase transition lines intersect at two tricritical points.

In the computer modeling of the critical behavior, both in semi-bounded systems and in films, it is assumed that the exchange interaction between the spins on the surface differs in terms of its value from the interaction in the bulk of a system. Here, it is not taken into account that the interaction between the surface spins and the first subsurface layer also differs from the bulk interaction. However, taking this effect into consideration may have a significant influence on the phase diagram of a system and the critical-behavior regime, since the bulk of a system and the surface play the role of an external field for each other in ordering. The value of the exchange integral between the surface spins and the first subsurface layer determines the intensity of their interaction. As it was shown in the paper [19] for semi-bounded antiferromagnetics, taking this effect into consideration changes the phase diagram of a system. Instead of two tricritical points, there is only one tetracritical point in the phase diagram.

The aim of this paper is to study by computer modeling the phase transitions in thin films, described by the antiferromagnetic Ising model, at different values of surface energy and values of the interaction of the surface with the subsurface layer.

\section{System description}

The Hamiltonian of the antiferromagnetic Ising model can be written down in the following form:

$$
H=-J_{B} \sum_{B} S_{i} S_{j}-J_{S} \sum_{S} S_{i} S_{j}-J_{S B} \sum_{S} S_{i} S_{j},
$$

where $S_{i}$ are the values of the spin in the $i$-th node $(+1 / 2$ or $-1 / 2)$. In the first summand, summing is performed for all the spin pairs of the nearest neighbors located not on the film surface. In the second summand, the pairs of the nearest spins located on one of the surfaces are summed. In the third summand, one spin in each pair is located on one of the surfaces, while the other spin is located in the nearest subsurface layer. As already mentioned in the introduction, the exchange integrals may differ, therefore let us introduce two parameters $-R_{S}=J_{S} / J_{B}$ and $R_{S B}=J_{S B} / J_{B}$.

In this work, we performed the computer modeling of thin films described by the antiferromagnetic Ising model with a cubic lattice, using the Monte Carlo method with the help of the Metropolis algorithm, widely used for the study of such systems [14]. The lattice had the linear dimensions $L \times L \times D$, where $D$ is the film thickness. The planes of free surface were specified by the equations $z=0$ and $z=D-1$. Along the axes $O X$ and $O Y$, periodic boundary conditions were used. To determine the phase transition temperature, the theory of finite-size scaling was applied [15].

To describe the antiferromagnetic phase transitions, let us introduce two order parameters. The order parameter $m$ will determine the antiferromagnetic order in the main bulk of a system and will be calculated as the staggered magnetization of the spins located not on the surface. The value of $m$ is equal to the difference between the magnetic moments of two sublattices. To study the surface phase transition, let us introduce the order parameter $m_{S}$, which is calculated as the staggered magnetization of the spins located on the free surface. The value of this order parameter was calculated only for one free surface specified by the equation $z=0$.

The critical temperature of transition was determined with the help of the bulk and surface Binder's fourth-order cumulants [14]:

$$
U=1-\frac{\left\langle m^{4}\right\rangle}{3\left\langle m^{2}\right\rangle^{2}}, \quad U_{S}=1-\frac{\left\langle m_{S}^{4}\right\rangle}{3\left\langle m_{S}^{2}\right\rangle^{2}} .
$$

The angle brackets denote thermodynamic averaging.

The phase transition temperatures were determined from the position of the intersection point of the Binder cumulants for systems with different dimensions of $L$. In order to find the bulk critical temperature $T_{N}$, the $U$ cumulants were used, and to determine the surface phase transition temperature $T_{S}$, the behavior of the $U_{S}$ cumulants was studied.

\section{Computer modeling results}

The computer experiment was performed for antiferromagnetic films having a thickness ranging from $D=4$ layers to $D=12$ layers. The values of the ratio between the exchange integrals varied from $R_{\mathrm{S}}=0.5$ to $R_{\mathrm{S}}=2.0$ with a step of 0.1 . For the second ratio between the exchange integrals, we took the limiting values $R_{S B}=1$ and $R_{S B}=R_{S}$. The value of the ratio between the exchange integrals has an influence on the surface energy of a system, which is composed of the energy of the interaction of the surface spins between each other and the energy of the interaction of the surface spins with the spins of the first subsurface layer. For all the values of the parameters, we calculated the Neel temperature $T_{N}$ of the bulk transition and the surface phase transition temperature $T_{S}$.

In the first place, it should be noted that in thin films the Neel temperature $T_{N}$ of the bulk phase transition depends on the film thickness and both ratios between the exchange integrals. This picture is essentially different from that in semi-bounded systems, where the Neel temperature of the bulk phase transition does not depend on the ratio between the exchange integrals and coincides with the corresponding value for unbounded systems. The dependence of the Neel temperature $T_{N}$ of the bulk phase transition on the ratio between the exchange integrals, $R_{S}$, at $R_{S B}=1$ for films of different thicknesses $D$ is shown in Fig. 1. Similar curves for $R_{S B}=R_{S}$ are shown in Fig. 2.

As it can be seen from Figs. 1 and 2, the bulk phase transition temperature grows with increasing $R_{s}$. This dependence can be easily accounted for by the influence of the surface energy on the whole system. This effect is absent in a semi-bounded system, since the surface makes an infinitely small contribution to the total energy of a system. In the case of thin films, two surface layers make up a considerable part of the system and have a significant influence on the behavior of its thermodynamic functions. A similar dependence was obtained for ferromagnetic thin films in the study [20]. From 


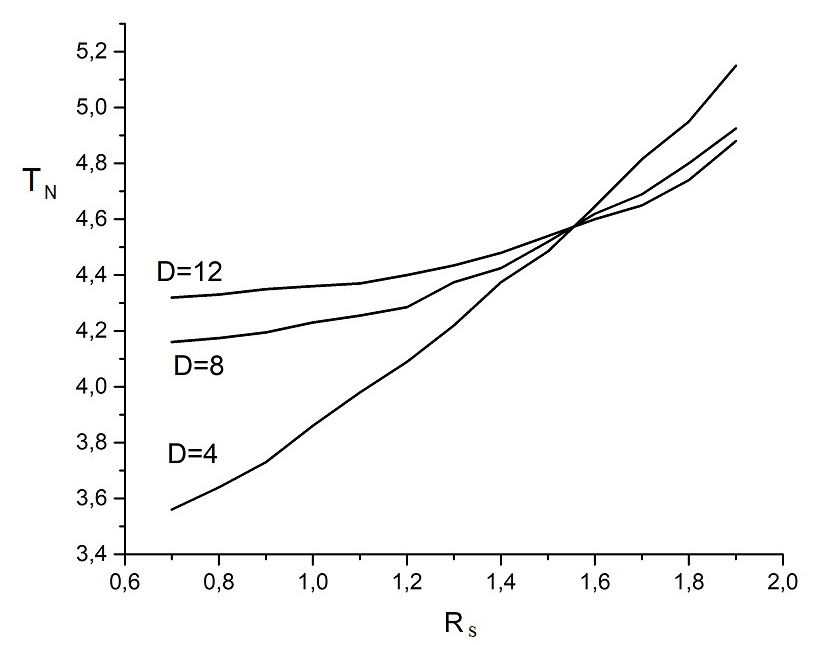

Fig 1. Dependence of the Neel temperature $T_{N}$ of the bulk phase transition on the ratio between the exchange integrals, $R_{S}$, at $R_{S B}=1$ for films with different thicknesses $D$.

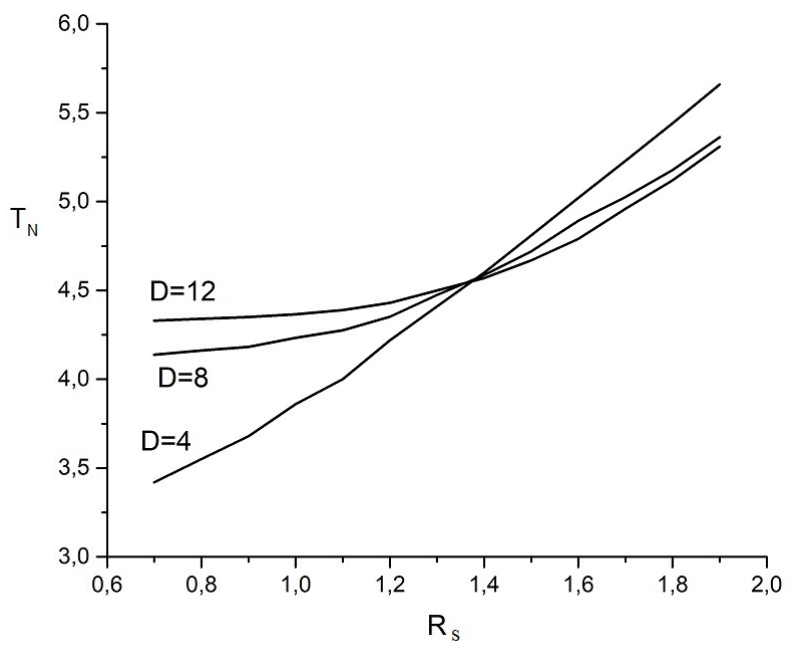

Fig 2. Dependence of the Neel temperature $T_{N}$ for the bulk phase transition on the ratio between the exchange integrals, $R_{S}$, at $R_{S B}=R_{S}$ for films with different thicknesses $D$.

the comparison of the curves in Figs. 1 and 2 it can be seen that the difference of the exchange integral of the interaction between the surface layer and the first subsurface layer leads to a more rapid growth of the phase transition temperature. In both figures there is an intersection point of the curves showing the dependence of the Neel temperature on the ratio between the exchange integrals for all the three dimensions of the system. The presence of these points is conditioned by the geometry of the system. The spins on the surface have five nearest neighbors, whereas in the main bulk of the system each spin has six nearest neighbors. The intersection point of the curves corresponds to the case when the value of the surface exchange integral compensates the absence of one nearest neighbor.
The SD/OB phase is not observed in thin films. The influence of the surface layer of spins on the whole system is substantial, therefore instead of two independent transitions, one transition with an intermediate temperature is observed. The tricritical point, to the right of which there is a surface phase transition, is characterized for thin films by a high temperature and large values of the ratio between the exchange integrals, $R_{S}$, which cannot be observed in actual systems. For instance, for $D=6$ the tricritical point is observed at $R_{S}=3.1$.

\section{Conclusion}

Thus, the critical behavior of thin antiferromagnetic films differs from the behavior of semi-bounded systems. In the first place, the bulk transition temperature is not constant, but grows with increasing surface energy. The surface phase transition can be observed only at large ratios between the exchange integrals, lying in a non-physical area.

\section{References}

1. M. I. Kaganov. JETP. 35(3), 631 (1972).

2. K. Binder. Phase transition and critical phenomena (Eds. Domb C. And Lebowitz J.L.). NY Academ. Press. (1983) V. 3. P. $325-331$.

3. H. W. Diehl. J. Mod. Phys. B. 11, 3503 (1997).

4. H. W. Diehl, M. Shpot. Nucl.Phys. B. 528, 595 (1998).

5. S. V. Belim. JETP. 103(4), 611 (2006).

6. S. V. Belim. JETP. 106(4), 773 (2008).

7. C. Rau, M. Robert. Phys. Rev. Lett. 58, 2714 (1987).

8. A.S. Kamzin, L.A. Grigor'ev. JETP Letters. 57(9), 552 (1993).

9. C.-Yu. Lin, J.-L. Li, Y.-H. Hsieh, K.-L. Ou, B. A. Jones. Phys. Rev. X. 2, 021012 (2012).

10. P. Ruiz-Diaz, T. R. Dasa, V.S. Stepanyuk. Phys. Rev. Lett. 110, 267203 (2013).

11. P. Ruiz-Diaz, V.S. Stepanyuk. J. Phys. D. Appl.Phys. 47, 105006 (2014).

12. O. O. Brovko, P. Ruiz-Dhaz, T.R. Dasa, V.S. Stepanyuk. J. Phys. Condens. Matter. 26, 093001 (2014).

13. I. Bernal-Villamil, S. Gallego Phys. Rev. B. 94, 075431 (2016).

14. D. P. Landau, K. Binder. Phys. Rev. B. 17, 2328 (1978).

15. C. Ruge, F. Wagner. Phys. Rev. B. 52, 4209 (1995).

16. M. Vendruscolo, M. Rovere, A. Fasolino. Europhys. Lett. 20, 547 (1992).

17. S. V. Belim, T. A. Koval'. Phys. Metals Metallogr. 115(9), 843 (2014).

18. S. V. Belim, T. A. Koval'. J. of Surface Investigation. 9(6), 1130 (2015).

19. S. V. Belim, E. V. Trushnikova. Journal of Physics: Conf. Series. 944, 012011 (2017).

20. M. Hamedoun, K. Bouslykhane, H. Bakrim, A. Hourmatallah, N. Benzakour, R. Masrour. J. Magn. Magn. Mater. 301, 22 (2006). 